\title{
SOME SEQUENCE SPACES AND ALMOST CONVERGENCE
}

\author{
SUDARSAN NANDA
}

(Received 24 March 1975; revised 16 September 1975)

\begin{abstract}
In this paper we investigate some new sequence spaces which naturally emerge from the concept of almost convergence. Just as ordinary, absolute and strong summability, it is expected that almost convergence must give rise to almost, absolutely almost and strongly almost summability. Almost and absolutely almost summable sequences have been discussed by several authors. The object of this paper is to introduce the spaces of strongly almost summable sequences which happen to be complete paranormed spaces under certain conditions. Some topological results, characterisation of strongly almost regular matrices, uniqueness of generalized limits and inclusion relations of such sequences have been discussed.
\end{abstract}

\section{Introduction}

Let $S$ be the set of all sequences real or complex and $l_{\infty}$ denote the Banach space of bounded sequences $x=\left\{x_{k}\right\}_{k=0}^{\infty}$ normed by $\|x\|=\sup _{k \geq 0}\left|x_{k}\right|$. Let $D$ be the shift operator on $S$, that is, $D x=\left\{x_{k}\right\}_{k=1}^{\infty}, D^{2} x=\left\{x_{k}\right\}_{k=2}^{\infty}$ and so on.

It may be recalled that [see Banach (1932)] Banach limit $L$ is a nonnegative linear functional on $l_{\infty}$ such that $L$ is invariant under the shift operator (that is, $\left.L(D x)=L(x) \forall x \in l_{\infty}\right)$ and that $L(e)=1$ where $e=\{1,1, \cdots\}$. A sequence $x \in l_{x}$ is called almost convergent [Lorentz (1948)] if all Banach limits of $x$ coincide. Let $\hat{c}$ denote the set of all almost convergent sequences. Lorentz (1948) proved that

$$
\hat{c}=\left\{x: \lim _{m \rightarrow \infty} \frac{1}{m+1} \sum_{i=0}^{m} x_{n+i} \text { exists uniformly in } n\right\} .
$$

The summability methods of real or complex sequences by infinite matrices are of three types [see Maddox (1970), p. 185] - ordinary, absolute and strong. In the same vein, it is expected that the concept of almost convergence must give rise to three types of summability methods-almost, absolutely almost and strongly almost. The almost summable sequences have been discussed by King (1966), Schaefer (1969) and some others. More recently Das and Nanda 
(unpublished) have considered absolute almost convergent and absolute almost summable sequences. Therefore our only concern is the strongly almost summable sequences, which naturally come up for investigation.

The strongly summable sequences have been systematically investigated by Hamilton and Hill (1938), Kuttner (1946) and some others. The spaces of strongly summable sequences were introduced and studied by Maddox (1967, 1970).

The purpose of this paper is to introduce the spaces of strongly almost summable sequences, which will fill up a gap in the existing literature.

Let $\boldsymbol{A}=\left(a_{n k}\right)$ be an infinite matrix of nonnegative real numbers and $p=\left\{p_{k}\right\}$ be a sequence such that $p_{k}>0$. (These assumptions are made throughout.) We write $A x=\left\{A_{n}(x)\right\}$ if $A_{n}(x)=\Sigma_{k} a_{n k}\left|x_{k}\right|^{p_{k}}$ converges for each $n$ (Here and afterwards summation without limits runs over 1 to $\infty$ ) and then we write

$$
t_{m, n}(x)=\frac{1}{m+1} \sum_{i=0}^{m} A_{n+i}(x)=\sum_{k} a(n, k, m)\left|x_{k}\right|^{p_{k}}
$$

where

$$
a(n, k, m)=\frac{1}{m+1} \sum_{i=0}^{m} a_{n+i, k} .
$$

We define [see Maddox (1967)],

$$
\begin{aligned}
{[A, p]_{0} } & =\left\{x: A_{n}(x) \rightarrow 0\right\} ; \\
{[A, p] } & =\left\{x: A_{n}(x-l e) \rightarrow 0 \text { for some } l\right\} ;
\end{aligned}
$$

and

$$
[A, p]_{\infty}=\left\{x: \sup _{n} A_{n}(x)<\infty\right\} .
$$

The spaces $[A, p]_{0},[A, p]$ and $[A, p]_{\infty}$ are respectively called the spaces of strongly summable to zero, strongly summable and strongly bounded sequences.

We now write

$$
\begin{aligned}
{[\hat{A}, p]_{0} } & =\left\{x: t_{m, n}(x) \rightarrow 0 \text { uniformly in } n\right\} ; \\
{[\hat{A}, p] } & =\left\{x: t_{m, n}(x-l e) \rightarrow 0 \text { for some } l \text { uniformly in } n\right\} ;
\end{aligned}
$$

and

$$
[\hat{A}, p]_{\infty}=\left\{x: \sup _{m, n} t_{m, n}(x)<\infty\right\}
$$


The sets $[\hat{A}, p]_{0},[\hat{A}, p]$ and $[\hat{A}, p]_{\infty}$ will be respectively called the spaces of strongly almost summable to zero, strongly almost summable and strongly almost bounded sequences.

If $x$ is strongly almost summable to $l$ we write $x_{k} \rightarrow l[\hat{A}, p]$. A pair $(A, p)$ will be called strongly almost regular if

$$
x_{k} \rightarrow l \Rightarrow x_{k} \rightarrow l[\hat{A}, p] .
$$

In Theorem 1 we obtain suitable conditions for the above sets to be complete linear topological spaces and in Theorems 2 and 3 we discuss $r$-convexity and locally boundedness for these spaces. Theorem 4 characterises strongly almost regular matrices. In Theorems 5 and 6 we consider uniqueness of generalised limits and inclusion relations for the strongly almost summable sequences.

\section{The main result}

We first establish a number of useful propositions.

Proposition 1. If $p \in l_{\infty}$, then $[\hat{A}, p]_{0},[\hat{A}, p]$ and $[\hat{A}, p]_{\infty}$ are linear spaces over $\mathbb{C}$.

Proof. We consider only $[\hat{A}, p]$. Others can be treated similarly. If $H=\sup p_{k}$ and $K=\max \left(1,2^{H-1}\right)$, we have [see Maddox (1967), p. 346].

$$
\left|a_{k}+b_{k}\right|^{p_{k}} \leqq K\left(\left|a_{k}\right|^{p_{k}}+\left|b_{k}\right|^{p_{k}}\right)
$$

and for $\lambda \in \mathbb{C}$,

$$
|\lambda|^{p_{k}} \leqq \max \left(1,|\lambda|^{H}\right) \text {. }
$$

Suppose that $x_{k} \rightarrow l[\hat{A}, p], y_{k} \rightarrow l^{\prime}[\hat{A}, p]$ and $\lambda, \mu \in \mathbb{C}$. Then we have

$$
t_{m, n}\left(\lambda x+\mu y-\left(\lambda l+\mu l^{\prime}\right) e\right) \leqq K K_{1} t_{m, n}(x-l e)+K K_{2} t_{m, n}\left(y-l^{\prime} e\right)
$$

where $K_{1}=\sup |\lambda|^{p_{k}}$ and $K_{2}=\sup |\mu|^{p_{k}}$, and this implies that $\lambda x+$ $\mu y \rightarrow\left(\lambda l+\mu l^{\prime}\right)[\hat{A}, p]$.

This completes the proof.

It is evident that

-

$$
\begin{aligned}
& {[A, p]_{0} \subset[\hat{A}, p]_{0},[A, p] \subset[\hat{A}, p],} \\
& {[A, p]_{\infty} \subset[\hat{A}, p]_{\infty}[\hat{A}, p]_{0} \subset[\hat{A}, p],} \\
& {[\hat{A}, p]_{0} \subset[\hat{A}, p]_{\infty} .}
\end{aligned}
$$

But we have been able to prove ' $[\hat{A}, p] \subset[\hat{A}, p]_{x}$ ' only under additional restrictions on $A$. 
We have

Proposition 2. $[\hat{A}, p] \subset[\hat{A}, p]_{\infty}$ if

$$
\|A\|=\sup _{m} \sum_{k} a(n, k, m)<\infty
$$

Proof. Suppose that $x_{k} \rightarrow l[\hat{A}, p]$ and (3) holds. Now by the inequality (1),

$$
\begin{aligned}
t_{m, n}(x) & =t_{m, n}(x-l e+l e) \\
& \leqq K t_{m, n}(x-l e)+K \sum_{k} a(n, k, m)|l|^{p_{k}} \\
& \leqq K t_{m, n}(x-l e)+K\left(\sup |l|^{p_{k}}\right) \sum_{k} a(n, k, m) .
\end{aligned}
$$

Therefore $x \in[\hat{A}, p]_{\infty}$ and this completes the proof.

Remark. Some known sequence spaces are obtained by specialising $A$ and therefore some of the results proved here extend the corresponding results obtained for the special cases.

Proposition 3. Let $p \in l_{\infty}$, then $[\hat{A}, p]_{0}$ and $[\hat{A}, p]_{\infty}\left(\inf p_{k}>0\right)$ are linear topological spaces paranormed by $g$ defined by

$$
g(x)=\sup _{m, n}\left[t_{m, n}(x)\right]^{1 / M}
$$

where $M=\max \left(1, H=\sup p_{k}\right)$. If (3) holds, then $[\hat{A}, p]$ has the same paranorm.

Proof. Clearly $g(0)=0$ and $g(x)=g(-x)$. Since $M \geqq 1$, by Minkowski's inequality it follows that $g$ is subadditive. We now show that the scalar multiplication is continuous. It follows from the inequality (2) that

$$
g(\lambda x) \leqq \sup |\lambda|^{p_{k} / M} g(x) .
$$

Therefore $x \rightarrow 0 \Rightarrow \lambda x \rightarrow 0$ (for fixed $\lambda$ ). Now let $\lambda \rightarrow 0$ and $x$ be fixed. Given $\varepsilon>0 \exists N$ such that

$$
t_{m, n}(\lambda x)<\varepsilon / 2(\forall n, \forall m>N)
$$

Since $t_{m, n}(x)$ exists for all $m$, we write

$$
t_{m, n}(x)=K(m),(1 \leqq m \leqq N)
$$

and

$$
\delta=\left(\frac{\varepsilon}{2 K(m)}\right)^{1 / p_{k}}
$$


Then for $|\lambda|<\delta$,

$$
t_{m, n}(\lambda x)<\varepsilon / 2(\forall n, 1 \leqq m \leqq N) .
$$

It follows from (5) and (6) that

$$
\lambda \rightarrow 0 \Rightarrow \lambda x \rightarrow 0 \text { ( } x \text { fixed). }
$$

This proves the assertion about $[\hat{A}, p]_{0}$.

If inf $p_{k}=\theta>0$ and $0<|\lambda|<1$, then $\forall x \in[\hat{A}, p]_{\infty}$,

$$
g^{M}(\lambda x) \leqq|\lambda|^{\theta} g^{M}(x)
$$

Therefore $[\hat{A}, p]_{\infty}$ has the paranorm $g$. If (3) holds it is clear from Proposition 2 that $g(x)$ exists for each $x \in[\hat{A}, p]$.

This completes the proof.

REMARK. It is evident that $g$ is not a norm in general. But if $p_{k}=p \forall k$, then clearly $g$ is a norm for $1 \leqq p<\infty$ and a $p$-norm for $0<p<1$.

Proposition 4. $[\hat{A}, p]_{0}$ and $[\hat{A}, p]_{\infty}$ are complete with respect to their paranorm topologies. $[\hat{A}, p]$ is complete if (3) holds and

$$
\sum_{k} a(n, k, m) \rightarrow 0 \text { uniformly in } n
$$

Proof. Let $\left\{x^{i}\right\}$ be a Cauchy sequence in $[\hat{A}, p]_{0}$. Then $\exists$ a sequence $x$ such that $g\left(x^{i}-x\right) \rightarrow 0(i \rightarrow \infty)$. Since $g$ is subadditive it follows that $x \in[\hat{A}, p]_{0}$.

The completness of $[\hat{A}, p]_{\infty}$ can be similarly obtained.

We now consider $[\hat{A}, p]$. If (3) holds and $\left\{x^{i}\right\}$ is a Cauchy sequence in $[\hat{A}, p]$, Then (as above) $\exists x$ such that $g\left(x^{i}-x\right) \rightarrow 0$. If (7) holds then from inequality (4) it is clear that $[\hat{A}, p]=[\hat{A}, p]_{0}$.

This completes the proof.

Combining the above facts we obtain our main result.

Theorem 1. Let $p \in l_{\infty}$. Then $[\hat{A}, p]_{0}$ and $[\hat{A}, p]_{\infty}\left(\right.$ inf $\left.p_{k}>0\right)$ are complete linear topological spaces paranormed by g. If (3) and (7) hold then $[\hat{A}, p]$ has the same property. If further $p_{k}=p \forall k$, they are Banach spaces for $1 \leqq p<\infty$ and $p$-normed spaces for $0<p<1$.

\section{Some topological results}

We now study locally boundedness and $r$-convexity for the spaces of strongly almost summable sequences. We start with some definitions.

For $0<r \leqq 1$ a non-void subset $U$ of a linear space is said to be absolutely 
$r$-convex if $x, y \in U$ and $|\lambda|^{r}+|\mu|^{r} \leq 1$ together imply that $\lambda x+\mu y \in U$. It is clear that if $U$ is absolutely $r$-convex, then it is absolutely $t$-convex for $t<r$. A linear topological space $X$ is said to be $r$-convex if every neighbourhood of $0 \in X$ contains an absolutely $r$-convex neighbourhood of $0 \in X$. The $r$-convexity for $r>1$ is of little interest, since $X$ is $r$-convex for $r>1$ if and only if $X$ is the only neighbourhood of $0 \in X$, [see Maddox and Roles (1969)]. A subset $B$ of $X$ is said to be bounded if for each neighbourhood $U$ of $0 \in X \exists$ an integer $N>1$ such that $B \subseteq N U . X$ is called locally bounded if there is a bounded neighbourhood of zero.

We first prove:

THEOREM 2. Let $0<p_{k} \leqq 1$. Then $[\hat{A}, p]_{\infty}$ and $[\hat{A}, p]_{\infty}$ are locally bounded if inf $p_{k}>0$. If (3) holds, then $[\hat{A}, p]$ has the same property.

Proof. We shall only prove for $[\hat{A}, p]_{\infty}$. Let inf $p_{k}=\theta>0$. If $x \in[\hat{A}, p]_{\infty}$, then $\exists$ a constant $K^{\prime}>0$ such that

$$
\sum_{k} a(n, k, m)\left|x_{k}\right|^{p_{k}} \leqq K^{\prime}(\forall m, n) .
$$

For this $K^{\prime}$ and given $\delta>0$ choose an integer $N>1$ such that

$$
N^{\theta} \geqq \frac{K^{\prime}}{\delta}
$$

Since $1 / N<1$ and $p_{k} \geqq \theta$ we have

$$
\frac{1}{N^{p_{k}}} \leqq \frac{1}{N^{\theta}}
$$

Then for all $m$ and $n$, we get

$$
\begin{aligned}
\sum_{k} a(n, k, m)\left|\frac{x_{k}}{N}\right|^{p_{k}} & \leqq \frac{1}{N^{\theta}} \sum_{k} a(n, k, m)\left|x_{k}\right|^{p_{k}} \\
& \leqq \frac{K^{\prime}}{N^{\theta}} \leqq \delta .
\end{aligned}
$$

Therefore by taking supremum over $m$ and $n$ we get,

$$
\left\{x: g(x) \leqq K^{\prime}\right\} \subseteq N\{x: g(x) \leqq \delta\} .
$$

For every $\delta>0 \exists N>1$ for which the above inclusion holds and so

$$
\left\{x: g(x) \leqq K^{\prime}\right\}
$$

is bounded.

This completes the proof. 
It is known that every locally bounded linear topological space is $r$-convex for some $r$ such that $0<r \leqq 1$. But the following theorem gives exact conditions for $r$-convexity.

THEOREM 3. Let $0<p_{k} \leqq 1$. Then $[\hat{A}, p]_{0}$ and $[\hat{A}, p]_{\times}$are $r$-convex for all $r$ where $0<r<\liminf p_{k}$. Moreover, if $p_{k}=p \leqq 1 \forall k$, then they are $p$-convex. [Â, $\left.p\right]$ has the same properties if (3) holds.

Proof. We shall prove the theorem only for $[\hat{A}, p]_{\infty}$. Let $x \in[\hat{A}, p]_{\infty}$ and $r \in\left(0, \liminf p_{k}\right)$. Then $\exists k_{0}$ such that $r \leqq p_{k}\left(\forall k>k_{0}\right)$. Now define

$$
\hat{g}(x)=\sup _{m, n}\left[\sum_{k=1}^{k_{0}} a(n, k, m)\left|x_{k}\right|^{r}+\sum_{k=k_{0+1}}^{\infty} a(n, k, m)\left|x_{k}\right|^{p_{k}}\right] .
$$

Since $r \leqq p_{k} \leqq 1\left(\forall k>k_{0}\right), \hat{g}$ is subadditive. Further for $0<|\lambda| \leqq 1$,

$$
|\lambda|^{p_{k}} \leqq|\lambda|^{r} \quad\left(\forall k>k_{0}\right)
$$

Therefore for such $\lambda$ we have

$$
\hat{g}(\lambda x) \leqq|\lambda|^{\prime} \hat{g}(x) .
$$

Now for $0<\delta<1$,

$$
U=\{x: \hat{g}(x) \leqq \delta\}
$$

is an absolutely $r$-convex set, for $|\lambda|^{r}+|\mu|^{r} \leqq 1$ and $x, y \in U$ imply that

$$
\begin{aligned}
\hat{g}(\lambda x+\mu y) & \leqq \hat{g}(\lambda x)+\hat{g}(\mu y) \leqq|\lambda|^{\prime} \hat{g}(x)+|\mu|^{\prime} \hat{g}(y) \\
& \leqq\left(|\lambda|^{r}+|\mu|^{r}\right) \delta \leqq \delta .
\end{aligned}
$$

If $p_{k}=p \forall k$, then for $0<\delta<1$,

$$
V=\{x: g(x) \leqq \delta\}
$$

is an absolutely $p$-convex set. This can be obtained by a similar analysis and therefore we omit the details.

This completes the proof.

REMARK. The conclusions of Theorems 2 and 3 also hold for the spaces of strongly summable sequences. The proofs are similar and therefore omitted. These results do not appear anywhere, although Maddox and Roles (1969) have obtained some results in some special cases.

\section{Some further results}

Let $X$ and $Y$ be two nonempty subsets of the space $S$ of sequences. If $x=\left\{x_{k}\right\} \in X$ implies that $\left\{\Sigma_{k} a_{n k} x_{k}\right\} \in Y$, we say that $A$ defines a (matrix) 
transformation from $X$ into $Y$, and we write $A: X \rightarrow Y$. $(X, Y)$ denotes the class of matrices $A$ such that $A: X \rightarrow Y$. Let $c_{0}$ and $\hat{c}_{0}$ respectively denote the linear spaces of null sequences and sequences almost convergent to zero.

We now characterise the class of strongly almost regular matrices.

THEOREM 4. Let $0<\theta \leqq p_{k} \leqq H<\infty$. Then $(A, p)$ is strongly almost regular if and only if $A \in\left(c_{0}, \hat{c}_{0}\right)$.

Remark. It is known that [see King (1966)] $A \in\left(c_{0}, \hat{c}_{0}\right)$ if and only if

(i) $\|\boldsymbol{A}\|<\infty$;

(ii) $\lim _{n \rightarrow \infty} a(n, k, m)=0$ uniformly in $n,(\forall k)$.

To prove Theorem 4 we require the following result.

LemMA 1. [see Maddox (1967), p. 347] If $p_{k}, q_{k}>0$, then

$$
c_{0}(q) \subset c_{0}(p) \Leftrightarrow \liminf \frac{p_{k}}{q_{k}}>0 .
$$

Proof of Theorem 4. Necessity. Suppose that $(A, p)$ is strongly almost regular. Therefore

$$
\left|x_{k}-l\right|^{1 / p_{k}} \rightarrow 0 \Rightarrow \sum_{k} a(n, k, m)\left|x_{k}-l\right| \rightarrow 0
$$

uniformly in $n$. Again since $1 / p_{k} \geqq 1 / H>0$, by Lemma 1

$$
x_{k} \rightarrow l \Rightarrow\left|x_{k}-l\right|^{1 / p_{k}} \rightarrow 0 .
$$

Thus

$$
x_{k} \rightarrow l \Rightarrow \sum_{k} a(n, k, m)\left(x_{k}-l\right) \rightarrow 0
$$

uniformly in $n$ and therefore $A \in\left(c_{0}, \hat{c}_{0}\right)$.

Sufficiency. Since $p_{k} \geqq \theta>0$, by Lemma 1 ,

$$
x_{k} \rightarrow l \Rightarrow\left|x_{k}-l\right|^{p_{k}} \rightarrow 0 \text {. }
$$

Again we have $A \in\left(c_{0}, \hat{c}_{0}\right)$. Therefore $x_{k} \rightarrow l[\hat{A}, p]$ and this concludes the proof.

Note that $p_{k} \leqq H$ is superfluous in the sufficiency and $\theta \leqq p_{k}$ is superfluous in the necessity.

We next consider the uniqueness of generalised limits.

TheOREM 5. Suppose that $A \in\left(c_{0}, \hat{c}_{o}\right)$ and $p=\left\{p_{k}\right\}$ converges to a positive limit. Then $x=\left\{x_{k}\right\} \rightarrow l \Rightarrow x_{k} \rightarrow l[\hat{A}, p]$ uniquely if and only if 


$$
\sum_{k} a(n, k, m) \not>0 \text { uniformly in } n \text {. }
$$

Proof. Necessity Suppose that $A \in\left(c_{0}, \hat{c}_{0}\right)$ and $\left\{p_{k}\right\}$ be bounded. Let $x_{k} \rightarrow l$ imply that $x_{k} \rightarrow l[\hat{A}, p]$ uniquely. We have $e \rightarrow 1[\hat{A}, p]$. Therefore the condition (8) must hold. For, otherwise $e \rightarrow 0[\hat{A}, p]$ which contradicts the uniqueness of $l$.

Note that the restriction on $\left\{p_{k}\right\}$ (except boundedness) is superfluous for the necessity.

Sufficiency. Suppose that the condition (8) holds and $A \in\left(c_{0}, \hat{c}_{0}\right)$ and that $p_{k} \rightarrow r>0$. Further assume that $x_{k} \rightarrow l$ implies $x_{k} \rightarrow l[\hat{A}, p]$ and $x_{k} \rightarrow l[\hat{A}, p]$ where $\left|l-l^{\prime}\right|=a>0$. Then we get

$$
\lim _{n \rightarrow \infty} \sum_{k} a(n, k, m) u_{k}=0 \quad \text { (uniformly in } n \text { ) }
$$

where

$$
u_{k}=\left|x_{k}-l\right|^{p_{k}}+\left|x_{k}-l^{\prime}\right|^{p_{k}} \text {. }
$$

By the assumption we have $u_{k} \rightarrow a^{r}$. Since $A \in\left(c_{0}, \hat{c}_{0}\right), u_{k} \rightarrow a^{r}$ implies that

$$
\sum_{k} a(n, k, m)\left|u_{k}-a^{\prime}\right| \rightarrow 0 \text { (uniformly in } n \text { ) }
$$

But we have

$$
\begin{aligned}
a^{r} \sum_{k} a(n, k, m) & \leqq \sum_{k} a(n, k, m) u_{k} \\
& +\sum_{k} a(n, k, m)\left|u_{k}-a^{r}\right|
\end{aligned}
$$

Now by (9), (10) and (11) it follows that

$$
\lim _{m \rightarrow \infty} \sum_{k} a(n, k, m)=0 \quad \text { (uniformly in } n \text { ). }
$$

Since this contradicts (8), we must have $l=l^{\prime}$. This completes the proof.

Suppose that $0<p_{k} \leqq q_{k}$. We conclude this note by showing that $[\hat{A}, q] \subset$ $[\hat{A}, p]$ is not true in general. However the inclusion holds for a special class. We prove

TheOREM 6. (a) Suppose that $\|A\|<\infty$ and $q_{k} / p_{k}$ is bounded, then $[\hat{A}, q] \subset[\hat{A}, p]$. (b) Let $A$ be an almost regular matrix and suppose that $q_{k} / p_{k} \rightarrow \infty$, then $[\hat{A}, q] \not \subset[\hat{A}, p]$.

Proof. (a) Write $w_{k}=\left|x_{k}-l\right|^{q_{k}}$ and $p_{k} / q_{k}=\lambda_{k}$. So that $0<\lambda \leqq \lambda_{k} \leqq 1(\lambda$ constant). Let $x \in[\hat{A}, q]$. Then 


$$
\sum_{k} a(n, k, m) w_{k} \rightarrow 0 \quad \text { (uniformly in } n \text { ) }
$$

Define $u_{k}=w_{k}\left(w_{k} \geqq 1\right),=0\left(w_{k}<1\right)$ and $v_{k}=0\left(w_{k} \geqq 1\right),=w_{k}\left(w_{k}<1\right)$. So that $w_{k}=u_{k}+v_{k}, w_{k^{k}}^{\lambda_{k}}=u_{k^{k}}^{\lambda^{k}}+v_{k^{k}}^{\lambda^{k}}$. Now it follows that $u_{k^{k}}^{\lambda^{k}} \leqq u_{k} \leqq w_{k}, v_{k^{k}}^{\lambda_{k}} \leqq v_{k}^{\lambda}$. We have the inequality [see Maddox (1967), p. 351]

$$
\sum_{k} a(n, k, m) w_{k^{k}}^{\lambda^{k}} \leqq \sum_{k} a(n, k, m) w_{k}+\left(\sum_{k} a(n, k, m) v_{k}\right)^{\lambda}\|A\|^{1-\lambda} .
$$

Therefore $x \in[\hat{A}, p]$ and this completes the proof.

(b) It is known that [see Duran (1972), corollary to Theorem 1] no matrix is both almost regular and almost schur. Therefore, if $\boldsymbol{A}: 3$ almost regular, it follows that there is always a sequence $y=\left\{y_{k}\right\}$ consisting of 0 's and 1's which is not almost $A$-summable. Consequently, $b=\left\{b_{k}\right\}$ defined by $b=(y+e) / 4$ is not almost $A$-summable. Define $x_{k}=b_{k}^{1 / p_{k}}$. Then $x_{k} \not \rightarrow 0[\hat{A}, p]$. But $x_{k} \rightarrow 0[\hat{A}, q]$ since $\left|b_{k}\right| \leqq \frac{1}{2}<1$ and $q_{k} / p_{k} \rightarrow \infty$. This completes the proof.

Finally, the author is grateful to Professor G. Das for suggestions and guidance and to the referee for some valuable comments.

\section{References}

S. Banach (1932), Theorie des Operations Lineaires (Warszawa).

J. P. Duran (1972), 'Infinite matrices and almost convergence', Math. Z. 128, 75-83.

G. Das and S. Nanda (to appear), Some sequence spaces and absolute almost convergence.

H. J. Hamilton and J. D. Hill (1938), 'On strong summability', Amer. J. Math. 60, 588-94.

B. Kuttner (1946), 'Note on strong summability', J. London Math. Soc. 21, 118-22.

J. P. King (1966), 'Almost summable sequences', Proc. Amer. Math. Soc. 17, 1219-25.

G. G. Lorentz (1948), 'A contribution to the theory of divergent sequences', Acta Math. 80, 167-190.

I. J. Maddox (1967), 'Spaces of strongly summable sequences', Quart. J. Math. Oxford Ser. (2) 18, 345-55.

I. J. Maddox and J. W. Roles (1969), 'Absolute convexity in certain topological linear spaces', Proc. Camb. Philos. Soc. 66, 541-45.

I. J. Maddox (1970), Elements of Functional Analysis (Camb. Univ. Press).

Paul Schaefer (1969), 'Almost convergent and almost summable sequences', Proc. Amer. Math. Soc., 20, 51-54.

Department of Mathematics, Regional Engineering College,

Rourkela 769008,

Orissa,

India. 